или небоскреб, и соответствующей строительной документации, подразумевают создание информации, доступной для двух типов аудитории - "покупателей" и "строителей". Покупателя больше интересуют планы этажей, трехмерные изображения, анимационные ролики и, конечно, расчет бюджета, строителя - полный перечень строительных документов и необходимых материалов. Чаще всего, программы САПР предоставляют только какой-либо один тип вышеупомянутой информации, и только ArchiCAD позволяет в одном файле интегрировать данные, понятные как профессионалу-строителю, так и покупателю. Эта особенность позволяет архитектору пользователю ArchiCAD сэкономить свое рабочее время и значительную часть средств своего клиента и поможет избежать возможных конфликтов еще до их возникновения[4].

Широко известная программа 3D MAX как программа для дизайнеров используется также и архитекторами. Это программное обеспечение для 3Dмоделирования, анимации и рендеринга, созданное и разработанное для игр и визуализации дизайна. Программа включена в коллекцию мультимедийных и развлекательных программных продуктов, предлагаемых Autodesk. Tрехмерная графика настолько прочно вошла в нашу жизнь, что мы сталкиваемся с ней, порой даже не замечая ее. Разглядывая интерьер комнаты на огромном рекламном щите, янтарный блеск льющегося нива в рекламном ролике, наблюдая, как взрывается самолет в остросюжетном боевике, многие не догадываются, что перед ними не реальные съемки, а результат работы мастера трехмерной графики. Область применения трехмерной графики необычайно широка. Использование компьютерных технологий при проектировании и разработке дизайна интерьера помогает увидеть конечный вариант задолго до того, как обстановка будет воссоздана.

Таким образом, программное обеспечение САПР представляет собой сложную программную систему, включающую в себя десятки и сотни компонентов. Для успешного функционирования и конкурентоспособности промышленных предприятий в современных условиях абсолютно необходимы передовые информационные технологий. Они позволяют не только решать широкий круг задач в сфере автоматизации финансово-хозяйственной и управленческой деятельности, но и осуществлять комплексную автоматизацию основных технологических и производственных бизнес-процессов.

$$
* * *
$$

1. Гулякин Д.В. Социально-информационная компетентность в структуре деятельности личности // Научные проблемы гуманитарных исследований. - 2009. - № 12. С. 36-39.

2. Гулякин Д. В. Роль информационно-коммуникационных технологий в формировании социальноинформационной компетентности // Современные информационные технологии. - 2009. - №10. c. 121-123.

3. Добрицына И.А. От постмодернизма - к нелинейной архитектуре: Архитектура в контексте современной философии и науки. - М.: Прогресс-Традиция, 2004.

4. Адамчик М.В. Архитектура. Краткий справочник. - Изд.: Харвест, $2004-624$ с.

\title{
Карасев И.Р. \\ Диспетчеризация решения задач в многопроцессорном вычислительном комплексе реального времени
}

Национальный исследовательский ядерный университет «МИФИ» (Россия, Москва)

doi: $10.18411 / 1 j-05-2021-12$

\section{Аннотация}

Повышение эффективности работы над задачами возможна путем объединения нескольких ЭВМ в один ИВК. Построение ИВК - это индивидуальный процесс для выполнения определенных задач. Преимущество его использования состоит в 
возможности параллельного выполнения нескольких задач, следовательно, происходит экономия времени. Однако построение ИВК дорогостояще и требует внимательного подхода с учетом необходимой производительности и бюджета. Главная задача - найти оптимальное значение между затратами (на оборудование, персонал, обслуживание и т.д.) и нужным быстродействием всей системы и каждой отдельной машиной.

Ключевые слова: информационно-вычислительный комплекс (ИВК), электронно-вычислительная машина (ЭВМ), структура, алгоритм, анализ, процессор, память, совмещение.

\section{Abstract}

Improving the efficiency of working on tasks is possible by combining several computers into one information and computing complex. Building an information and computing complex is an individual process for performing certain tasks. The advantage of using it is the possibility of parallel execution of several tasks, therefore, there is a saving of time. However, building an information and computing complex is expensive and requires a careful approach, taking into account the required performance and budget. The main task is to find the optimal value between the costs (for equipment, personnel, maintenance, etc.) and the desired speed of the entire system and each individual machine.

Keywords: information and computing complex, electronic computer (computer), structure, algorithm, analysis, processor, memory, combination.

\section{1. Сглаживание требований к производительности и памяти.}

Часто случается, что при рассмотрении по тактам выполняемой задачи можно заметить, что в некоторых частях идет более высокая нагрузка, которую можно уменьшить и освободить ресурсы для того, чтобы не переплачивать за более дорогое оборудование, часть ресурсов из которого просто не будет использоваться.

С этой задачей помогает справиться сглаживание требований к производительности, которое, если это возможно, распределяет нагрузку между всей системой. Для того, чтобы выполнить сглаживание необходима какая-то задача, и таблица с исходными данными характеристик оборудования. Далее необходимо построить временную диаграмму, которую впоследствии необходимо переделать в таблицу требуемой производительности. Далее используя результаты составленной таблицы, необходимо произвести сглаживание требований к производительности.

Далее нужно построить таблицы занятости памяти, необходимую для оценки требуемого объема памяти.

Необходимо провести анализ занятости ячеек оперативной памяти. Для минимизации занятого объема памяти можно применить метод преобразования таблицы. При построении таблицы занятости памяти следует учитывать необходимость хранения входных и выходных данных, а также данных, предназначенных для использования другими задачами. Эту информацию можно получить из граф-схемы подсистемы или из ее матрицы смежности и из анализа показателей внешней и внутренней связности алгоритмов.

Первой таблицей будет «предварительная» таблица занятости памяти, где будут показаны входные, внутренние и выходные данные. После ее построения, данные сводятся в обобщенную таблицу, где на каждый такт будет загрузка суммарно равная всему объему данных на каждой цепочке и каждом такте.

Имея обобщенную таблицу, можно приступить к сглаживанию требований к памяти, для минимизации ресурсов задачи. Путем сдвига задач ищем более оптимальный вариант.

После проделанной работы мы можем оценить, насколько нам удалось уменьшить требования к производительности и памяти для задачи. 


\section{2. Планирование параллельных вычислительных процессов.}

Для решения задачи планирования параллельных вычислительных процессов необходимо выполнить следующие действия:

1. Сформировать параллельные цепочки путем преобразований графсхемы алгоритмов. Эта работа выполняется на начальных этапах разработки ИВК при декомпозиции алгоритмического комплекса.

2. Оценить степень параллелизма, т.е. число параллельных цепочек в принятой для реализации на проектируемом ИВК алгоритмической подсистеме. Эта характеристика легко определяется по временной диаграмме.

3. Разработать временную диаграмму для каждой параллельной цепочки.

4. Рассчитать требуемую производительность и объем памяти по каждой цепочке и установить требования к процессорам.

Следует оценить величину производительности П для максимальных требований к производительности, а затем рассматривать возможность решения на нем нескольких цепочек задач, требующих меньшей производительности.

Пусть $\widetilde{\Pi}_{l}=\max _{1 \leq i \leq I}\left\{\widetilde{\Pi}_{i}\right\}$, где I - количество параллельных цепочек в граф схеме алгоритмов, или степень параллелизма.

Возьмем процессор с производительностью $\Pi=\alpha \widetilde{\Pi}_{i}$, где $\alpha$ коэффициент пропорциональности, $\widetilde{\Pi}_{i}$ производительность і-ого процессора. Эффективность использования его производительности при решении задач 1-ой цепочки $\mathrm{u}=\frac{\widetilde{\Pi}_{l}}{\Pi}=\frac{1}{\alpha}<1$,

Поскольку при выборе процессора был предусмотрен запас производительности. При решении задач любой другой $\mathrm{r}-и ̆(\mathrm{r} \neq 1)$ цепочки $u_{r}=\frac{\widetilde{\Pi}_{r}}{\Pi}=\frac{\widetilde{\Pi}_{r}}{\widetilde{\Pi}_{l} \alpha}<u_{l}$

Это означает, что на всех цепочках, кроме 1-й (у которой нагрузка максимальна), запасы производительности при использовании процессора, одинакового с выбранным для 1-й цепочки, могут составлять значительную величину. Это позволяет использовать его для решения нескольких параллельных цепочек задач. Планирование загрузки в этом случае можно осуществить несколькими способами:

1. Полное совмещение без преобразования временных диаграмм;

2. Полное совмещение с преобразованием временных диаграмм;

3. Частичное совмещение;

Следует учитывать, что совмещение выполнения команд увеличивает объем оборудования и усложняет схемы управления тем сильнее, чем больше число уровней совмещения.

Для того, чтобы добиться наибольшей производительности при меньших тратах, необходимо рассмотреть структуры информационно-вычислительного комплекса.

\section{3. Выбор структуры информационно-вычислительного комплекса.}

Выделим 4 структуры:

1. Простейшая структура ИВК - одномашинная (с одним вычислителем).

2. Многомашинный децентрализованный вычислительный комплекс со связями через устройства обмена.

3. Централизованная структура ИВК

4. Централизованная структура с общей оперативной памятью.

После определения параметров технических средств, остается выбрать структуру, которая вносит окончательную определенность в требования к отдельным модулям.

Рассмотрим различные варианты выводов, которые могут быть сделаны:

a) Заданным требованиям удовлетворяет одномашинный вариант на основе серийных технических средств. При этом обязательно должно быть проверено соответствие этих средств заданным условиям 
эксплуатации; несоответствие приводит к необходимости разработки новой техники

b) Заданные требования можно обеспечить в структурах разных типов на основе серийных ЭВМ, причем для каждого типа существует несколько вариантов. Каждый вариант отличается по количеству ЭВМ или модулей и требованиям к их характеристикам, причем число устройств невелико: $2 \leq \mathrm{K} \leq 5$.

c) Расчет производительности для серийных средств приводит к значениям К больше 5.

По имеющимся выводам рассчитываем полную стоимость производства различных структур и выявляем более подходящую.

Для каждой из них производится расчет стоимости их построения и работы с ней.

Если сделан вывод о возможности применения варианта а), то такой вариант выбирается, однако при проверке условия П> некоторый запас производительности и памяти (20-30\%) для возможной модернизации системы.

Если сделан вывод о применении варианта b), то прежде всего следует оценить затраты. Для серийных ЭВМ или модулей их стоимости $\mathrm{S}$ известны и указаны в ценниках и прейскурантах. Затраты на эксплуатацию обычно рассчитывают по формуле:

$$
S_{\text {экспл }}=\mathrm{c}_{\ni} k_{\ni}+\mathrm{c}_{p} k_{p}+\mathrm{c}_{3} k_{3},
$$

где $\mathrm{c}_{\ni}+\mathrm{c}_{p}+\mathrm{c}_{3}-$ стоимости энергии, ремонта и зарплата обслуживающего персонала соответственно; $k_{\ni}+k_{p}+k_{3}-$ коэффициенты пропорциональности.

Если сделан вывод о применении варианта с), то в этом случае решение можно принять только после анализа целесообразности разработки новых технических средств. Такая целесообразность может быть подтверждена только экономическими расчетами с учетом сроков разработки и фактора времени. Трудность заключается в том, что, естественно, соответствующих точных данным для проектируемых устройств нет и приходиться использовать аппроксимации по малому числу точек и эмпирические оценки. Кроме того, возникает новая задача расчета рационального количества основных устройств ИВК.

$$
* * *
$$

1. Я.А. Хетагуров, Ю.Г. Древс «Проектирование информационно-вычислительных комплексов»: Учеб. для вузов по спец. "АСУ". - М.: Высш. шк., 1987. - 280 с.

2. Гордеев А.В. «Операционные системы. 2-е изд.»/ СПб.: Питер, 2004. - 415c.

3. Бурдонов И. Б., Косачев А. С., Пономаренко В. Н. «Операционные системы реального времени»/ Институт системного программирования РАН, 2006. - 98с.

4. Жмакин А. П. «Архитектура ЭВМ: 2-е изд.», перераб. и доп.: учеб. пособие. - СПб.: БХВПетербург, 2010. - 352 с

Колесников Р.A.

Жанровая классификация музыки с помощью акустических признаков

Российский технологический университет МИРЭА (Россия, Москва)

doi: $10.18411 / 1 j-05-2021-13$

\section{Аннотация}

В статье рассмотрены аудио признаки, которые наиболее часто используются в анализе и классификации аудио и представлен план разработки и краткий пример жанрового классификатора на языке программирования Python.

Ключевые слова: аудиоанализ, аудио, классификация аудио, нейронные сети. 